\title{
Author Correction: Ultrafast and highly sensitive infrared photodetectors based on two-dimensional oxyselenide crystals
}

Jianbo Yin (1) 1, Zhenjun Tan ${ }^{1,2}$, Hao Hong ${ }^{3}$, Jinxiong Wu' ${ }^{1}$, Hongtao Yuan ${ }^{4}$, Yujing Liu ${ }^{1}$, Cheng Chen ${ }^{5}$, Congwei Tan', Fengrui Yao ${ }^{3}$, Tianran Li ${ }^{1}$, Yulin Chen ${ }^{5}$, Zhongfan Liu ${ }^{1,2}$, Kaihui Liu (D) ${ }^{2,3}$ \& Hailin Peng ${ }^{1,2}$

Correction to: Nature Communications https://doi.org/10.1038/s41467-018-05874-2, published online 17 August 2018.

The original version of this Article contained an error in Fig. $1 \mathrm{~g}$ in which a top x-axis was shown with incorrect labels for the wavelength. The correct version of Fig. 1g, which removes this labelling, is:

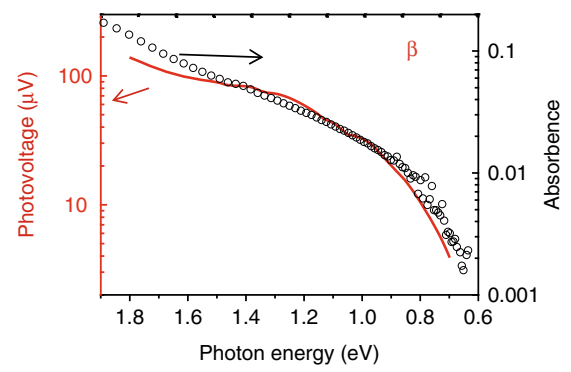

which replaces the previous incorrect version:

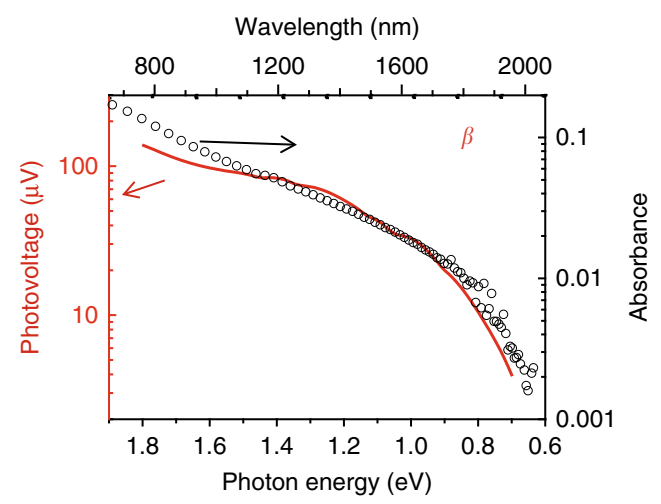

This has been corrected in both the PDF and HTML versions of the Article.

\footnotetext{
${ }^{1}$ Center for Nanochemistry, Beijing Science and Engineering Centre for Nanocarbons, Beijing National Laboratory for Molecular Sciences, College of Chemistry and Molecular Engineering, Peking University, Beijing 100871, China. ${ }^{2}$ Academy for Advanced Interdisciplinary Studies, Peking University, Beijing 100871, China. ${ }^{3}$ State Key Laboratory for Mesoscopic Physics, School of Physics, Peking University, Beijing 100871, China. ${ }^{4}$ National Laboratory of SolidState Microstructures, College of Engineering and Applied Sciences, and Collaborative Innovation Center of Advanced Microstructures, Nanjing University, Nanjing 210093, China. ${ }^{5}$ Clarendon Laboratory, Department of Physics, University of Oxford, Parks Road, Oxford OX1 3PU, UK. These authors contributed equally: Jianbo Yin, Zhenjun Tan, Hao Hong, Jinxiong Wu. Correspondence and requests for materials should be addressed to K.L. (email: khliu@pku.edu.cn) or to H.P. (email: hlpeng@pku.edu.cn)
} 
Published online: 29 July 2019

(c) (i) Open Access This article is licensed under a Creative Commons Attribution 4.0 International License, which permits use, sharing, adaptation, distribution and reproduction in any medium or format, as long as you give appropriate credit to the original author(s) and the source, provide a link to the Creative Commons license, and indicate if changes were made. The images or other third party material in this article are included in the article's Creative Commons license, unless indicated otherwise in a credit line to the material. If material is not included in the article's Creative Commons license and your intended use is not permitted by statutory regulation or exceeds the permitted use, you will need to obtain permission directly from the copyright holder. To view a copy of this license, visit http://creativecommons.org/licenses/by/4.0/.

(C) The Author(s) 2019 